\title{
L(t, 1)-Colouring of Wheel Graphs
}

\author{
P. Pandey ${ }^{1}$, J. V. Kureethara ${ }^{2 *}$ \\ ${ }^{1}$ Dept. of Mathematics and Statistics, Christ University, Bengaluru, India \\ ${ }^{2 *}$ Dept. of Mathematics and Statistics, Christ University, Bengaluru, India \\ *Corresponding Author: frjoseph@christuniversity.in, Tel.: +91-93410-94110
}

Available online at: www.isroset.org

Received 13/Oct/2017, Revised 29/Oct/2017, Accepted 5/Nov/2017, Online 30/Dec/2017

\begin{abstract}
An L(t, 1)-Colouring of graph is the colouring of the vertices of a graph with non negative integers such that the vertices which are adjacent to each other receives colour with their colour difference not belonging to set $T$ with integers including 0 and the vertices which are at distance 2 gets distinct colours. This is a type of channel assignment problem. Allotting frequencies to the radio channels in a region is determined by non-overlapping nature of the transmissions. The $\mathrm{L}(\mathrm{t}$, 1)-Colouring takes the inspiration from the famous T-Colouring and $\mathrm{L}(\mathrm{h}, \mathrm{k})$-Colouring of graphs. Both are celebrated colouring schemes. The $\mathrm{L}(\mathrm{t}, 1)$-span of the graph is the minimum of the highest colour used to colour the vertices of a graph out of all the possible $\mathrm{L}(\mathrm{t}, 1)$-colourings. We study the $\mathrm{L}(\mathrm{t}, 1)$-span of wheel graphs with respect a set $\mathrm{T}$ with consecutive integers and a set $\mathrm{T}$ whose elements are in AP with common difference d.
\end{abstract}

\section{Keywords-L(t, 1)-colouring, Communication networks, Channel assignment, Radio frequency, Colour span, Wheel Graphs}

\section{INTRODUCTION}

Graph colouring finds its use in the optimal assignment of radio frequencies to radio stations. The first kind of channel assignment problem was brought into the picture by $\mathrm{B}$. H. Metzger [1]. The T-colouring problem was one of the first colouring problems introduced by W. K. Hale [2] which put a constraint on the colour assignment of adjacent vertices. Later, F. Roberts [3] in his private communication with J. Griggs proposed that the disturbance in the transmission of signals is not only due to neighbouring transmitters but also due to the transmitters at distance 2 . This led to the study of distance two labelling. Labelling vertices of graphs at distance 2 was studied extensively by J. R. Griggs and R. K. Yeh[4].

We introduced an $\mathrm{L}(\mathrm{t}, 1)$-colouring which finds its foundation in T-colouring and $\mathrm{L}(2,1)$-colouring[5].

In this work, we study the bounds for $\mathrm{L}(\mathrm{t}, 1)$-span of wheel graph. All standard definitions and notations related to graphs are according to [6].

The paper is organized as follows. Section I contains the introduction to $\mathrm{L}(\mathrm{t}, 1)$-Colouring and Section II contains the related work. Section III is about the methodology. Section IV has the major results and illustrations. The $\mathrm{L}(\mathrm{t}, 1)$ Colouring of wheel graph for a set $\mathrm{T}$ with consecutive integers and a set $\mathrm{T}$ whose elements are in AP with common difference $\mathrm{d}$ form the major part of the study in this section.
Section IV concludes the research work indicating future directions.

\section{RELATED WORK}

The concept of $\mathrm{L}(\mathrm{t}, 1)$-Colouring was introduced in [5]. Bounds for $\mathrm{L}(\mathrm{t}, 1)$-span of stars, multipartite graph and cycles have already been studied in [5] and [9].

\section{Methodology}

There are three theorems in Section IV. We use proof by exhaustion and combinatorial arguments to prove the theorems. Illustrations are also supplied.

\section{RESUltS AND DISCUSSION}

Definition 4.1 A wheel graph $W_{n}$ of order $\mathrm{n}$, sometimes simply called an $n$-wheel, is a graph that contains a cycle of order $\mathrm{n} 1$, and for which every graph vertex in the cycle is connected to one other graph vertex (which is known as the hub)[7].

\section{A. $L(t, 1)$-Colouring of wheel graph for set $T$ with consecutive integers}

Definition 4.2 For a given set $\mathrm{T}$ with consecutive integers from 0 to $\mathrm{r}$, where $\max T=r$, the $\mathrm{L}(\mathrm{t}, 1)$-span of wheel graph is same as the $\mathrm{L}(\mathrm{h}, \mathrm{k})$-span of wheel graph with $h=r+1$ and $k=1$. 
Since wheel graph is also a Halin graph, in 2007 Zhang and $\mathrm{Ma}[8]$ studied the bound for Halin graph and gave $\lambda(G ; h ; k) \leq(2 k-1) \Delta+6 h 4 k 1$. This gives us the $\mathrm{L}(\mathrm{t}$, 1)-span of wheel graph as $\lambda_{t, 1}\left(W_{n}\right) \leq(\mathrm{n}-1)+6(\mathrm{r}+1)-$ 5.

The set $\mathrm{T}$ plays an important role in $\mathrm{L}(\mathrm{t}, 1)$-colouring. Due to randomness in the structure of set $\mathrm{T}$, we find the bound for $\mathrm{L}(\mathrm{t}, 1)$-span of wheel graph for set $\mathrm{T}$ where elements of set $\mathrm{T}$ are in arithmetic progression.

\section{B. $L(t, 1)$-Colouring of wheel graph where elements of set $T$ are in AP with common difference d}

Theorem 4.1 For set $T$ whose elements are in arithmetic progression with first term 0 and common difference $d=2$, the $L(t, 1)$-span of wheel graph is given by

$$
\lambda_{t, 1}\left(W_{n}\right)= \begin{cases}r+\left\{d\left\lceil\frac{n-2}{2}\right]\right\} & \text { for } n<2|T| \\ n+(r-1) & \text { for } n \geq 2|T|\end{cases}
$$

where $\max \{T\}=r$.

Proof. Since the wheel graph has diameter 2, all the vertices of the graph will get distinct colours. Let the vertices of the wheel be labelled as $\mathrm{v}_{1}, \mathrm{v}_{2}, \ldots, \mathrm{v}_{\mathrm{n}-1}, \mathrm{v}_{\mathrm{n}}$ with $\mathrm{v}_{1}$ being the central vertex and $v_{2}, v_{3}, \ldots, v_{n}$ being vertices on the cycle of the wheel.

Case 1: $n<2|T|$

Since the central vertex is the vertex with the maximum degree, we colour the central vertex with 0 (the least colour available). All the other vertices in the graph are at distance one from the central vertex. The colours assigned to them cannot belong to set $\mathrm{T}$. This implies that some of them can get the colours missing from set $\mathrm{T}$. We colour the vertex $\mathrm{v}_{2}$ by 1 (the first missing colour from the set). Vertex $v_{2}$ cannot get any odd colour since the difference of two odd number is even which is part of set T. All the vertices on the cycle of the wheel which are at distance one from the central vertex. So $\mathrm{v}_{2}$ should get an even number not in set T. i.e., $r+2$. Vertex $\mathrm{v}_{3}$ being adjacent to the vertex with colour $r+2$ can be assigned odd number not in set T. i.e., the second missing colour. Thus all vertices with even position (vertices $v_{2 n}$ ) can get colours missing from set $\mathrm{T}$ and all vertices with odd positions (vertices $\mathrm{v}_{2 \mathrm{n}+1}$ ) can get colours $r+n d$ for all $\mathrm{n} \geq 1$. Thus the required bound is obtained.

Case $2: n \geq 2|T|$

We can colour the vertices till $\mathrm{v}_{2|\mathrm{~T}|-1}$ using the above method. Vertices after $\mathrm{V}_{2|\mathrm{~T}|-1}$ can be coloured using consecutive colours after $r+(|T|-1) d$. Thus $\mathrm{v}_{\mathrm{n}}$ gets the colour $n+(r-1)$.

\section{Illustration 1}

For a set $\mathrm{T}=\{0,2,4,6,8,10\}$, the $\mathrm{L}(\mathrm{t}, 1)$-Colouring of the wheel with a number of vertices 5 is in Figure 1.

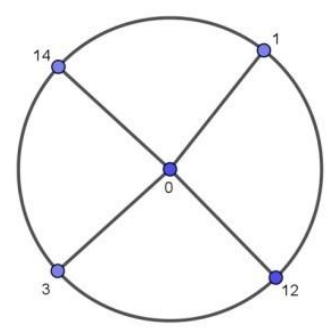

Figure 1. $\mathrm{L}(\mathrm{t}, 1)$-colouring of $\mathrm{W}_{5}$

$\mathrm{L}(\mathrm{t}, 1)$-span, in this case, is 14 which is in accordance with the result obtained above.

Theorem 4.2 For set $T$ whose elements are in arithmetic progression with first term 0 and common difference $d>3$, the $L(t, 1)$-span of wheel graph is given by

$$
\begin{gathered}
\lambda_{t, 1}\left(W_{n}\right) \begin{cases}<r & \text { for } n \leq[(d-1)(|T|-1)]+1 \\
=r+2 & \text { for } n=[(d-1)(|T|-1)]+2 \\
=(p-1)+r & \text { for } n>[(d-1)(|T|-1)]+2\end{cases} \\
\text { where } p=\{n-[(d-1)(|T|-1)]\} \text { and } \max \{T\}=r .
\end{gathered}
$$

Proof. We prove the theorem in three cases. Let the vertices of the wheel be labelled as $\mathrm{v}_{1}, \mathrm{v}_{2}, \ldots, \mathrm{v}_{\mathrm{n}}{ }^{-1}, \mathrm{v}_{\mathrm{n}}$ with $\mathrm{v}_{1}$ being the central vertex and $v_{2}, v_{3}, \ldots v_{n}$ being vertices on the cycle of the wheel. Let $c$ be the function which assigns colours to vertices of the graph. For a set $\mathrm{T}$ with $\mathrm{n}$ elements of form $\mathrm{a}_{0}$, $\mathrm{a}_{0}+\mathrm{d}, \mathrm{a}_{0}+2 \mathrm{~d}, \ldots, \mathrm{a}_{0}+(\mathrm{n}-1) \mathrm{d}$. L(t, 1)-span of the wheel can be obtained in following cases.

Case $1: n \leq[(d-1)(|T|-1)]+1$

The central vertex can be coloured using colour 0 . All the other vertices in the graph are at a distance one from the central vertex. Therefore, the colours assigned to them cannot belong to set $\mathrm{T}$. If set $\mathrm{T}$ has a common difference of $\mathrm{d}$ $>3$, there will be colours missing from set $\mathrm{T}$. We can assign the missing colours to the vertices of the cycle of wheel graph since these missing colours will differ by either 1 or 2 . Since $\mathrm{d}$ considered here is greater than 3 , we can assign missing colours to the vertices of the cycle of wheel consecutively except for the wheels with $\mathrm{n}$ of the form $(d-1) m+2$ where $m=1,2, \ldots$ In this case, we cannot assign the last consecutive colour to $\mathrm{v}_{\mathrm{n}}$ since $\mid c\left(v_{2}\right)-$ $c\left(v_{n}\right) \mid \in T$. Therefore, we assign colour to the last vertex by skipping the consecutive colour and giving it the next colour. This will lead to $\left|c\left(v_{n}\right)-c\left(v_{n-1}\right)\right|=3 \notin T$, since $d>3$. Thus $\mathrm{L}(\mathrm{t}, 1)$-span span of the wheel is always the less than $r$.

Case 2: $n=[(d-1)(|T|-1)]+2$

In this case, we colour the wheel in a similar way as in case one. Therefore, vertex $\mathrm{v}_{\mathrm{n}-1}$ gets colour $r-1$. Thus, the last vertex cannot be assigned colour $\mathrm{r}$ or $r+1$ since $\mid c\left(v_{n}\right)-$ 
$c\left(v_{1}\right) \mid=r \in T$ and since $\left|c\left(v_{n}\right)-c\left(v_{2}\right)\right|=r \in T$. Thus only $r+2$ is the least colour which can be assigned. Hence, we have the result.

Case 3: $n>[(d-1)(|T|-1)]+2$

We colour the vertices in a similar way as in case 1 till $\mathrm{v}_{[\mathrm{d} \text { - }}$ 1)(|T|-1)]+1. Next vertices will get the colours $r+1, r+2, \ldots$ and so on consecutively till $[n-[(d-1)(|T|-1)]-1]+r$.

It is the least possible colouring since all the missing colours are exhausted in this process and colours less than the assigned colours will violate the condition for $\mathrm{L}(\mathrm{t}, 1)$ colouring.

\section{Illustration 2}

For a set $\mathrm{T}=\{0,5,10,15,20\}$, the $\mathrm{L}(\mathrm{t}, 1)$-Colouring of the wheel with a number of vertices 9 is in Figure 2.

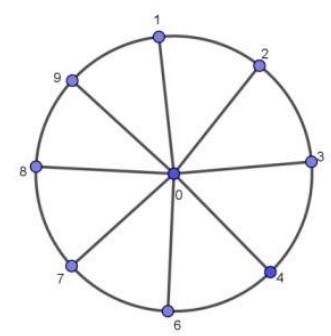

Figure 2. $\mathrm{L}(\mathrm{t}, 1)$-colouring of $\mathrm{W}_{9}$

$\mathrm{L}(\mathrm{t}, 1)$-span, in this case, is 9 which is in accordance with the result obtained above.

For $d=3$ the result remains same as in theorem 4.2 except for case $n=[(d-1)(|T|-1)]+2$. In this case $\left|c\left(v_{n}\right)-c\left(v_{n-1}\right)\right|=3 \in T$ if $r+2$ is assigned to $\mathrm{v}_{\mathrm{n}}$. Therefore, $r+3$ has to be assigned. Thus we get,

Theorem 4.3 For set $T$ whose elements are in arithmetic progression with first term 0 and common difference $d=3$, the $L(t, 1)$-span of wheel graph is given by

$\lambda_{t, 1}\left(W_{n}\right) \begin{cases}<r & \text { for } n \leq[(d-1)(|T|-1)]+1 \\ =r+3 & \text { for } n=[(d-1)(|T|-1)]+2 \\ =(p-1)+r & \text { for } n>[(d-1)(|T|-1)]+2\end{cases}$

where $p=\{n-[(d-1)(|T|-1)]\}$ and $\max \{T\}=r$.

\section{E. Illustration 3}

For a set $\mathrm{T}=\{0,3,6,9,12,15\}$, the $\mathrm{L}(\mathrm{t}, 1)$-Colouring of the wheel with a number of vertices 9 is in Figure 3.

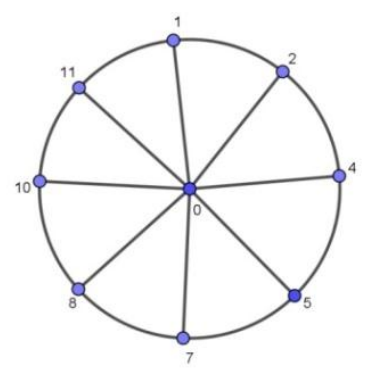

Figure 3. $\mathrm{L}(\mathrm{t}, 1)$-colouring of $\mathrm{W}_{9}$

$\mathrm{L}(\mathrm{t}, 1)$-span, in this case, is 11 which is in accordance with the result obtained above.

\section{CONCLUSION and Future Scope}

In this paper, we found the bounds for $L(t, 1)$-span of wheel graphs for set $\mathrm{T}$ with elements in the form of arithmetic progression. Since set $\mathrm{T}$ considered for the study is finite, it is easy to find the set of all gap numbers. Further, $\mathrm{L}(\mathrm{t}, 1)$ span of wheel graph by identifying the gap set i.e., numbers missing from set $\mathrm{T}$ can be studied.

\section{REFERENCES}

[1] B. H. Metzger, "Spectrum management technique," in 38th National ORSA meeting, Detroit, MI, US, 1970.

[2] W. K. Hale, "Frequency assignment: Theory and applications," in "Proc. IEEE", Vol. 68, pp. 1497-1514, 1980.

[3] F. S. Roberts, "T-colorings of graphs: recent results and open problems," "Discrete Mathematics", Vol. 93, pp. 229-245, 1991.

[4] R. K. Yeh, "Labelling graphs with a condition at distance two," $\mathrm{Ph}$. D. Thesis, University of South Carolina, 1990.

[5] P. Pandey, J. V. Kureethara, "L(t, 1)-colouring of graphs," arXiv: 1711.03096.

[6] D. B. West, "Introduction to Graph Theory", 2nd ed. Prentice Hall, US, 2001.

[7] F. Harary, "Graph Theory", Addison-Wesley, US, 1969.

[8] S. Zhang and Q. Ma, "Labelling of some planar graphs with a condition at distance two," Journal of Applied Mathematics and Computing, Vol. 24, pp. 421-426, 2007.

[9] P. Pandey, J. V. Kureethara, "L(t, 1)-colouring of cycles", in ICCTCEEC, Mysore, India, pp. 185-190, 2017.

\section{AUTHORS PROFILE}

Priyanka Pandey pursued BSc (Mathematics) from Govt. M. H. College of Home Science and Science for Women, Jabalpur, Madhya Pradesh affiliated to Rani Durgavati University of Jabalpur and MSc (Mathematics) from Christ University, Bengaluru, India. She is currently working as an Assistant Professor in Department of Mathematics and

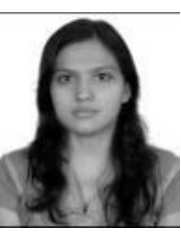
Statistics, Christ University, Bengaluru, India. She has interests in the areas of Combinatorics and Number Theory.

Joseph Varghese Kureethara received his $\mathrm{PhD}$ in Mathematics from MS University, Tirunelveli, India in 2010. He has MSc Mathematics and MA Economics from Madras University. $\mathrm{He}$ is currently an Associate Professor in the Department of Mathematics, Christ University, Bangalore,

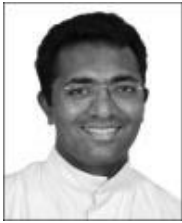


India. He is the Mathematics issue editor of Mapana Journal of Sciences and reviewer of many Mathematics journals. He is the author forty-eight articles in the fields of Graph Theory, Number Theory, Church History, Sacred Liturgy and Sports both in English and Malayalam. He is an active blogger and his blog has a total of more than 110 thousand pageviews. He co-edited two books. 